\title{
Unmasking the hidden tuberculosis mortality burden in a large post mortem study in Maputo Central Hospital, Mozambique
}

\author{
Alberto L. Garcia-Basteiro 1,2,3,13, Juan Carlos Hurtado (10) 2,4,13, \\ Paola Castillo $\mathbb{1}^{2,5,13}$, Fabiola Fernandes ${ }^{6}$, Mireia Navarro ${ }^{4}$, Lucilia Lovane ${ }^{6}$, \\ Isaac Casas ${ }^{4}$, Llorenc Quintó ${ }^{2}$, Dercio Jordao ${ }^{6}$, Mamudo R. Ismail $^{6}$, \\ Cesaltina Lorenzoni ${ }^{6,7}$, Carla Carrilho ${ }^{6}$, Ariadna Sanz $\mathbb{1}^{2}$, Natalia Rakislova ${ }^{2,5}$, \\ Aurea Mira ${ }^{8}$, Miriam J. Alvarez-Martínez ${ }^{2,4}$, Anélsio Cossa ${ }^{1}$, Frank Cobelens ${ }^{3}$, \\ Inácio Mandomando 1,9, Jordi Vila ${ }^{2,4}$, Quique Bassat ${ }^{1,2,10,11,12,14}$, \\ Clara Menendez $1,2,12,14$, Jaume Ordi ${ }^{2,5,14}$ and Miguel J. Martínez $2,4,14$
}

@ERSpublications

This study shows the usefulness of molecular assays in ascertaining TB diagnosis at death. It questions the information of clinical diagnoses obtained from hospital registries as a reliable tool for TB mortality estimation. http://bit.ly/2KrzTBJ

Cite this article as: Garcia-Basteiro AL, Hurtado JC, Castillo P, et al. Unmasking the hidden tuberculosis mortality burden in a large post mortem study in Maputo Central Hospital, Mozambique. Eur Respir J 2019; 54: 1900312 [https://doi.org/10.1183/13993003.00312-2019].

ABSTRACT Sensitive tools are needed to accurately establish the diagnosis of tuberculosis (TB) at death, especially in low-income countries. The objective of this study was to evaluate the burden of TB in a series of patients who died in a tertiary referral hospital in sub-Saharan Africa using an in-house real time PCR (TB-PCR) and the Xpert MTB/RIF Ultra (Xpert Ultra) assay.

Complete diagnostic autopsies were performed in a series of 223 deaths (56.5\% being HIV-positive), including 54 children, 57 maternal deaths and 112 other adults occurring at the Maputo Central Hospital, Mozambique. TB-PCR was performed in all lung, cerebrospinal fluid and central nervous system samples in HIV-positive patients. All samples positive for TB-PCR or showing histological findings suggestive of TB were analysed with the Xpert Ultra assay.

TB was identified as the cause of death in 31 patients: three out of 54 (6\%) children, five out of 57 (9\%) maternal deaths and 23 out of $112(21 \%)$ other adults. The sensitivity of the main clinical diagnosis to detect TB as the cause of death was $19.4 \%$ (95\% CI 7.5-37.5) and the specificity was 97.4\% (94.0-99.1) compared to autopsy findings. Concomitant TB (TB disease in a patient dying of other causes) was found in 31 additional cases. Xpert Ultra helped to identify 15 cases of concomitant TB. In 18 patients, Mycobacterium tuberculosis DNA was identified by TB-PCR and Xpert Ultra in the absence of histological TB lesions. Overall, 62 (27.8\%) cases had TB disease at death and 80 (35.9\%) had TB findings.

The use of highly sensitive, easy to perform molecular tests in complete diagnostic autopsies may contribute to identifying TB cases at death that would have otherwise been missed. Routine use of these tools in certain diagnostic algorithms for hospitalised patients needs to be considered. Clinical diagnosis showed poor sensitivity for the diagnosis of TB at death. 


\section{Introduction}

Tuberculosis (TB) remains a major public health concern in most countries of the world. In 2017, the World Health Organization (WHO) estimated that $\sim 10$ million new cases and 1.6 million deaths attributable to TB [1]. As a single cause of death (CoD), TB is the main infectious killer at a global level. In addition, it is the most frequent cause of HIV-associated deaths [2], and ranks among the principal CoD among females of reproductive age [3-5]. Similar to other countries in the region, the HIV and TB epidemic in Mozambique is devastating [1,6]. The estimated national incidence rate of TB in 2017 was 551 per 100000 inhabitants with a case fatality ratio of $31 \%(\sim 49000$ deaths in 2017) and HIV co-infection rate of $40 \%$ among new TB cases [1].

Accurate and reliable TB mortality data are fundamental to improve patient management, prioritise public health interventions and assess progress in the WHO End TB strategy indicators [7]. Despite the enormous TB burden, there is considerable uncertainty as to the actual mortality attributable to this disease, especially in some low-income countries with high disease burden [8]. Mortality estimates based on case fatality rates reported by national TB programmes are of low quality [9]. Clinical diagnosis and verbal autopsies have limited sensitivity and specificity for diagnosing $\mathrm{TB}$ as the CoD compared to complete diagnostic autopsy (CDA), the current gold standard [2, 9-13]. Studies assessing clinicopathological discrepancies have shown a high degree of misclassification when assigning deaths to $\mathrm{TB}$ in either direction (clinically missed TB causing death and false attribution of TB as the CoD when not present) $[10,11]$.

Additionally, most of the TB disease identified in CDA studies is considered to be responsible for the death of the patients [2]. The diagnosis of TB disease in these studies is based on obvious macroscopic disease, confirmed microscopically by the presence of granulomas with Ziehl-Neelsen stain-positive bacilli. However, it is likely that earlier forms of TB, in which pathological findings might not be that obvious, are missed. When there are other plausible causes of death and concomitant TB disease, the exact role that TB might have played in the chain of events leading to death may not be clear. This can be especially true in immunocompromised HIV-positive patients [14].

As part of a large post-mortem study conducted at a tertiary referral hospital in Mozambique [15-18], two molecular tests were used to diagnose TB, an in-house real-time PCR and the Xpert MTB/RIF Ultra assay (Xpert Ultra). The study had two main objectives: 1) to describe the overall burden of TB as the CoD and as a concomitant finding (TB disease identified at death, but not directly causing death) and 2) to assess the proportion of clinically missed TB cases.

\section{Methods}

\section{Study design}

This was an ancillary study to a prospective observational post mortem evaluation aimed at validating minimally invasive autopsy for CoD determination in different age groups compared with CDA, the gold standard technique [19]. This study included childhood ( $\geqslant 1$ month to 15 years), adult and maternal deaths (the latter defined as deaths among females while pregnant or within 42 days of termination of pregnancy) [20]. Traumatic deaths were excluded. The study was conducted from November 2013 to March 2015 at the Department of Pathology of the Maputo Central Hospital (Mozambique), a 1500-bed government-funded tertiary healthcare centre, in collaboration with the departments of paediatrics, internal medicine and obstetrics and gynaecology. Prior informed consent was obtained from the relatives of the deceased. The study received approval from the clinical research ethics committee of the Hospital Clinic of Barcelona (Spain; file 2013/8677) and the National Bioethics Committee of Mozambique (Mozambique; ref. 342/CNBS/13).

Affiliations: ${ }^{1}$ Centro de Investigação em Saúde de Manhiça (CISM), Maputo, Mozambique. ${ }^{2}$ ISGlobal, Hospital Clínic - Universitat de Barcelona, Barcelona, Spain. ${ }^{3}$ Amsterdam Institute for Global Health and Development, Amsterdam University Medical Centers, Amsterdam, The Netherlands. ${ }^{4}$ Dept of Microbiology, Hospital Clinic, Universitat de Barcelona, Barcelona, Spain. ${ }^{5}$ Dept of Pathology, Hospital Clinic, Universitat de Barcelona, Barcelona, Spain. ${ }^{6}$ Dept of Pathology, Faculty of Medicine/Eduardo Mondlane University and Maputo Central Hospital, Maputo, Mozambique. ${ }^{7}$ Ministry of Health - National Cancer Control Programme, Mozambique. ${ }^{8}$ Biomedical Diagnostic Centre (CDB), Hospital Clínic, University of Barcelona, Barcelona, Spain. ${ }^{9}$ Instituto Nacional de Saúde (INS), Ministério da Saúde, Maputo, Mozambique. ${ }^{10}$ ICREA, Catalan Institution for Research and Advanced Studies, Barcelona, Spain. ${ }^{11}$ Pediatric Infectious Diseases Unit, Pediatrics Dept, Hospital Sant Joan de Déu (University of Barcelona), Barcelona, Spain. ${ }^{12}$ Consorcio de Investigación Biomédica en Red de Epidemiología y Salud Pública (CIBERESP), Madrid, Spain. ${ }^{13}$ Contributed equally to this work and share primary authorship. ${ }^{14}$ Contributed equally to this work and share senior authorship.

Correspondence: Miguel J. Martínez, Dept of Microbiology, Hospital Clinic, Universitat de Barcelona, Spain, Barcelona Institute for Global Health, Spain. E-mail: myoldidclinic.cat 
The pathological and microbiological methods of the CDA procedures have been described elsewhere [21, 22], Samples were obtained from all organs for histological and microbiological analysis. Samples for microbiological testing were collected in nucleic-acid preserving buffer (ATL lysis buffer; Qiagen, Hilden, Germany). Clinical information was collected from each patient using a standardised questionnaire after thorough revision of the entire medical record. The data obtained included demographic data, past medical history, as well as information about the inpatient admission process, signs and symptoms, physical examination, laboratory and imaging results when available and treatment received during hospitalisation. For maternal deaths, the obstetric history was reviewed in addition. Following analyses of the CDA samples, a panel composed of a pathologist, a microbiologist and a clinician (paediatrician, internist or gynaecologist, depending on the age group) evaluated the pathological and microbiological reports of the CDA and the clinical data and assigned the CDA diagnosis of CoD. The main results of this validation project have been published [15-18].

HIV status was confirmed post mortem by an automated method detecting antibodies against HIV (ADVIA Centaur HIV 1/0/2 Enhanced assay; Siemens Healthcare Diagnostics, Tarrytown, NY, USA) and by viral load testing using the Cobas TaqMan HIV-1 test v2.0 (Roche Molecular Systems, Branchburg, NJ, USA).

\section{TB testing strategy and laboratory procedures}

The TB testing strategy of the study is summarised in figure 1. The initial microbiological diagnosis of TB was performed using an in-house real-time PCR targeting Mycobacterium tuberculosis (TB-PCR). TB-PCR was performed in all lung samples obtained at CDA, independently of the presence or absence of histological lesions, and in any other organ showing histological lesions suggestive of TB (granulomatous inflammatory reaction and/or caseous necrosis). In addition, in HIV-positive patients, TB was routinely tested by TB-PCR in all central nervous system and cerebrospinal fluid (CSF) samples (independently of the presence or absence of lesions). Histological testing for TB included Ziehl-Neelsen staining when TB was suspected on the haematoxylin and eosin-stained slides.

In addition, the Xpert MTB/RIF Ultra assay (hereafter Xpert Ultra) was performed in 1) any organ with histological lesions suggestive or compatible with TB and positive TB-PCR (cases with TB disease) and 2) in all deaths in which there was microbiological-histological discordance (histological lesions suggestive of TB with negative TB-PCR or positive TB-PCR without histological lesions suggestive of TB).

Tissue samples for Xpert Ultra were thawed and homogenised using a hand-held rotor-stator homogeniser (Qiagen) in ATL lysis buffer (Qiagen). $100 \mu \mathrm{L}$ of the homogenised tissue sample was added to $300 \mu \mathrm{L}$ of saline solution. The resulting $400 \mu \mathrm{L}$ of sample were mixed with $1600 \mu \mathrm{L}$ of Xpert Ultra Sample Reagent and then loaded into the cartridge. In house TB-PCR was performed using the procedures described by ESPASA et al. [23].

\section{Definitions of TB associated findings}

"Histological lesions suggestive of TB" were defined as granulomatous inflammatory reactions with or without caseous necrosis, independently of the presence or absence of visible acid-fast bacilli on ZiehlNeelsen staining. "Histological lesions compatible with TB" included nonspecific inflammatory reactions (such as neutrophilic inflammation) which have been described in TB [24].

“TB disease at death" included histological lesions suggestive of TB with a positive TB-PCR or a positive Xpert Ultra in any sample or when there were TB-compatible histological lesions and both TB-PCR and Xpert Ultra were positive. TB disease at death was further classified as "TB as CoD" when the review of the entire CDA (including histological, microbiological and clinical data) following a previously described algorithm of $\mathrm{CoD}$ determination, deemed TB to be the $\mathrm{CoD}$ [15], which corresponds to the "-a diagnosis" in the WHO international form of medical certificate of death [25]. A case was classified as "concomitant TB" when histological lesions compatible with TB were present at death, but the review of the CDA deemed another disease as the most likely CoD (“-b" or "-c" diagnoses or "other significant conditions" in the WHO international form of medical certificate of death). Finally, a case was classified as " $M$. tuberculosis DNA detection" when both TB-PCR and Xpert Ultra were positive in the absence of compatible histological findings. A single positive TB-PCR without any histological finding compatible with TB was considered a false-positive result, since sample contamination or a true false-positive result due to assay- or human-related performance could not be ruled out.

\section{Statistical analysis}

All the clinical data and the results of the histopathological and microbiological examination of the samples were analysed using Stata 13 (StataCorp, College Station, TX, USA). We calculated the proportions of 1) cases in which TB was the CoD;2) cases with concomitant TB; and 3) cases with 


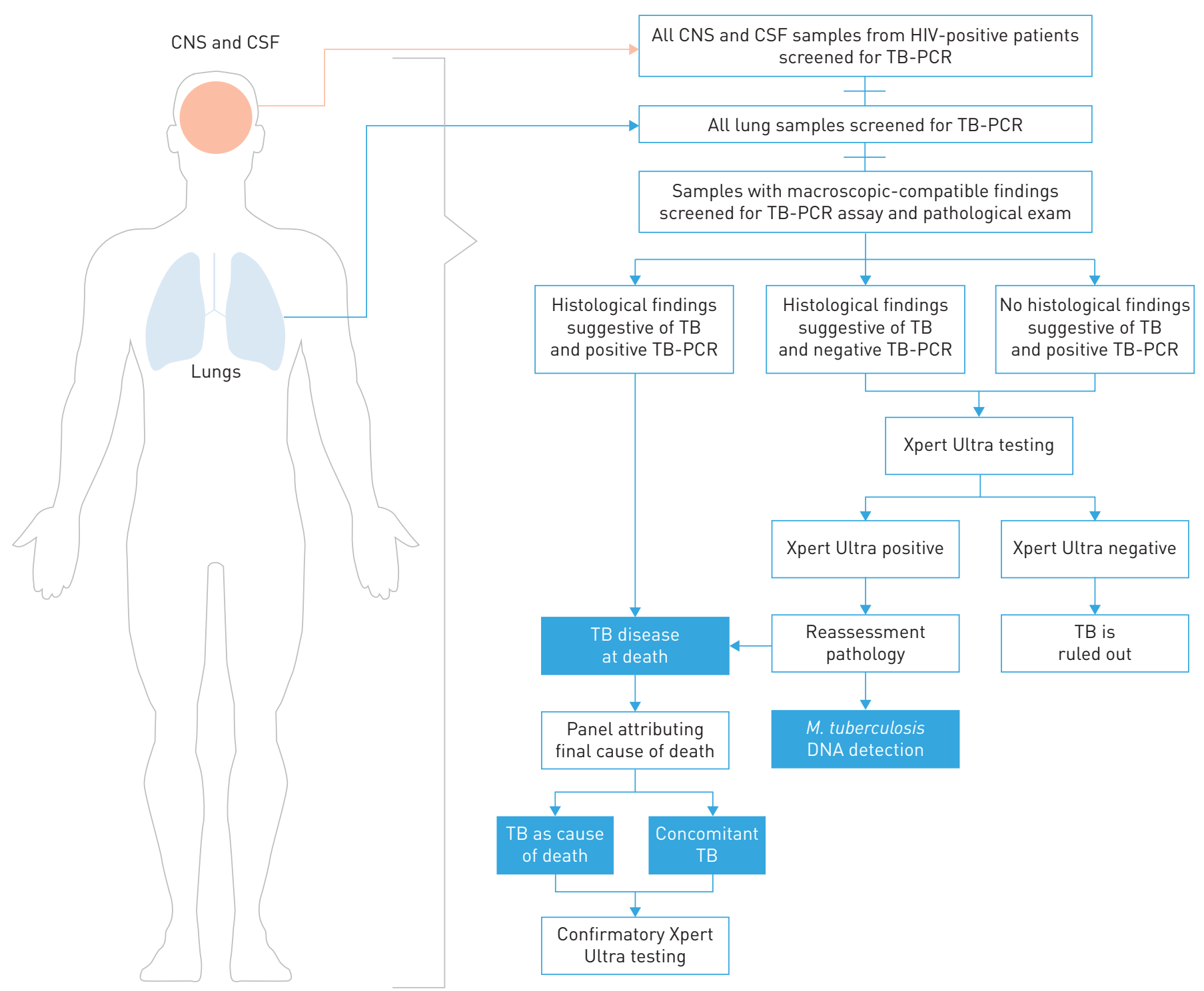

FIGURE 1 Algorithm for determination of tuberculosis (TB) diagnosis used in samples from complete diagnostic autopsies. CNS: central nervous system; CSF: cerebrospinal fluid; M. tuberculosis: Mycobacterium tuberculosis.

M. tuberculosis DNA. We estimated the sensitivity, specificity and predictive values of the clinical diagnoses to detect $\mathrm{TB}$ disease as the $\mathrm{CoD}$ (when the clinician specified $\mathrm{TB}$ as the main diagnosis as well as to detect concomitant TB (when the clinician specified TB among the diagnoses or initiated anti-TB treatment), using CDA diagnoses as the gold standard.

\section{Results}

The analysis included 223 deaths: 54 children, 57 maternal deaths and 112 other adults. HIV infection was identified in $32.7 \%$ (17 out of 52), 65.2\% (36 out of 57) and $63.2 \%$ (73 out of 112) of these deaths, respectively ( $56.5 \%$ of the overall series).

\section{TB-associated findings}

TB was diagnosed as the $\mathrm{CoD}$ in 31 patients: three out of 54 children (5.6\%, 95\% CI 1.2-15.4\%), five out of 57 maternal deaths $(8.8 \%, 95 \%$ CI $2.9-19.3 \%)$ and 23 out of 112 other adults $(20.5 \%, 95 \%$ CI 13.5-29.2\%). Among HIV-positive cases, TB was identified as the CoD in none out of 17 children (0\%), three out of $34(8.8 \%, 95 \%$ CI $1.9-23.7 \%)$ maternal deaths and 18 out of 73 (24.6\%, 95\% CI $15.3-36.1 \%)$ other adults. The most frequent form of TB as the CoD was miliary TB (23 out of $31 ; 74.2 \%)$, followed by pulmonary $\mathrm{TB}$ (six out of $31 ; 19.4 \%$ ) and TB meningitis (two out of $31 ; 6.5 \%$ ) (table 1 ). Among the 31 patients who died of TB, Xpert Ultra tested positive in all lung samples and 14 CSF samples. 
TABLE 1 Number of patients with tuberculosis (TB) disease as the cause of death (CoD), with concomitant TB disease and with Mycobacterium tuberculosis detection at death by study group and among HIV-positive cases.

\begin{tabular}{|c|c|c|c|c|}
\hline Autopsies & TB as CoD" & Concomitant TB & Total & $\begin{array}{l}\text { M. tuberculosis } \\
\text { DNA detection }\end{array}$ \\
\hline
\end{tabular}

\begin{tabular}{|c|c|c|c|c|c|c|}
\hline \multicolumn{7}{|l|}{ All autopsies } \\
\hline Children & 54 & $3(5.6)$ & $8(14.8)$ & $11(20.4)$ & $3(5.6)$ & $14(25.9)$ \\
\hline Adults & 112 & $23(20.5)$ & $18(16.1)$ & $41(36.6)$ & $10(8.9)$ & $51(45.5)$ \\
\hline \multicolumn{7}{|c|}{ Autopsies among HIV-positive cases } \\
\hline Children $^{f}$ & 17 & $0(0.0)$ & $3(17.6)$ & $3(17.6)$ & $0(0.0)$ & $3(17.6)$ \\
\hline Maternal deaths & 36 & 5 (13.9) & $4(11.1)$ & $9(25.0)$ & $5(13.9)$ & 14 (38.9) \\
\hline \multicolumn{7}{|c|}{$\begin{array}{l}\text { Data are presented as } n \text { or } n(\%) . ~ " \# \text { : HIV status of two cases could not be ascertained; }{ }^{\Uparrow}: \text { when review of the entire complete diagnostic autops } \\
\text { (CDA) (including histological, microbiological and clinical datal following a previously described algorithm of CoD determination deemed TB to } \\
\text { be the CoD. This definition required the presence of histological TB-compatible lesions and microbiological confirmation of TB by molecula } \\
\text { assays; }{ }^{+} \text {: when histological lesions compatible with TB were present at death, but review of the CDA deemed another disease to be the mos } \\
\text { likely cause. This definition required the presence of histological TB-compatible lesions and microbiological confirmation of TB by molecula } \\
\text { assays; }{ }^{\S} \text { : attributed when both TB-PCR and Xpert Ultra were positive in the absence of compatible histological findings; }{ }^{f} \text { : all cases in which } \\
\text { TB disease was found at death las the cause of death or as a concomitant findingl and cases in which only M. tuberculosis DNA were detected. }\end{array}$} \\
\hline
\end{tabular}

Concomitant TB was identified in 31 additional patients: eight out of 54 (14.8\%) children, 18 out of 112 (13.9\%) adults and five out of 57 (8.8\%) maternal deaths. Of these, 22 had pulmonary TB, five disseminated TB (more than one organ involved) and four had extrapulmonary TB involving a single organ (one case with splenic TB, three with TB meningitis). Table 2 and figure 2 show the $\mathrm{CoD}$ of the cases with concomitant TB. Initially, 15 out of 31 (48.4\%) cases of concomitant TB disease did not have histological lesions suggestive of TB. Only after Xpert Ultra results were available (which prompted further histological review) were the histological findings deemed as TB compatible lesions (mostly inflammatory lesions).

18 additional cases fulfilled the definition of $M$. tuberculosis DNA detection: three out of 54 (5.6\%) children, five out of 57 (8.8\%) maternal deaths and 10 out of $112(8.9 \%)$ adults. Of these cases, six died of an infectious cause and 12 of non-infectious conditions. Of the 18 cases in which only $M$. tuberculosis DNA was detected, six (33.3\%) were HIV-positive, and one (5.6\%) had a history of past TB.

Overall, TB findings were confirmed in 80 cases: 14 out of 54 (25.9\%) children, 15 out of 57 (26.3\%) maternal deaths and 51 out of $112(45.5 \%)$ other adults. Among HIV-positive patients, TB was confirmed in three out of 17 (17.6\%), 14 out of $36(38.9 \%)$ and 37 out of $74(50.7 \%)$ of patients in each study group, respectively (table 1). Rifampicin resistance was detected by Xpert Ultra in eight cases.

\section{Clinical characteristics of patients with TB findings}

A clinical history of TB was reported in none of the children, in two (3.5\%) maternal deaths and in $16(14.3 \%)$ adults. 20 out of $31(64.5 \%)$ patients with TB as the CoD had reported cough compared to nine out of $31(29 \%)$ among those with concomitant TB at death $(\mathrm{p}=0.005)$. Among patients with TB findings, eight cases were on anti-TB treatment prior to admission, and in 10 cases anti-TB treatment was initiated during admission. No differences were observed in terms of fever at admission, between patients with $\mathrm{TB}$ as the $\mathrm{CoD}$ and those with concomitant $\mathrm{TB}$ (17 out of 31 (55\%) versus 19 out of 31 (61\%), $\mathrm{p}=0.6$ ). The characteristics of cases with $\mathrm{TB}$ disease as the $\mathrm{CoD}$, with concomitant $\mathrm{TB}$, with $M$. tuberculosis DNA detection and those without any TB finding are shown in table 3.

\section{Clinicopathological discrepancies}

Assuming that the first (main) clinical diagnosis was the CoD for clinicians, they had considered TB disease as the $\mathrm{CoD}$ in 11 of the 223 patients (4.9\%). However, the clinical diagnosis of TB as the CoD was correct in only six patients (figure 2). Thus, the main clinical diagnosis had a sensitivity to detect $\mathrm{TB}$ disease as CoD of $19.4 \%$ (six out of $31,95 \%$ CI $7.5-37.5 \%$ ) and a specificity of $97.4 \%$ (95\% CI 94.0 99.1\%) (table 4). Among the six cases in which clinicians correctly specified TB as the main clinical diagnosis, complete agreement with the form or localisation was only confirmed in two cases of 
TABLE 2 Main cause of death of patients with concomitant tuberculosis (TB)

\begin{tabular}{|c|c|c|c|c|c|}
\hline & Group & HIV status & CDA A diagnosis & Infectious agent identified & Type of TB (organs affected) \\
\hline 1 & Children & Negative & Rabies & Rabies virus & Pulmonary \\
\hline 2 & Children & Negative & Peritonitis & Unspecified & Pulmonary \\
\hline 3 & Children & Negative & Malignant brain tumour & & Pulmonary \\
\hline 5 & Children & Negative & Meningoencephalitis & Cryptococcus gattii & Extrapulmonary (CNS) \\
\hline 6 & Children & Positive & Meningitis & Streptococcus pneumoniae & Pulmonary \\
\hline 7 & Children & Positive & Pneumocystosis & Pneumocystis jirovecii & Extrapulmonary (CNS) \\
\hline 10 & Maternal deaths & Positive & Septic abortion & Mycoplasma hominis & Pulmonary (miliary, both lungs) \\
\hline 11 & Maternal deaths & Positive & Encephalitis & Unspecified & Pulmonary \\
\hline 12 & Maternal deaths & Positive & Puerperal sepsis & & Pulmonary \\
\hline 13 & Maternal deaths & Negative & Pneumonia & Unspecified & Pulmonary \\
\hline 14 & Other adults & negative & Mucormycosis & Rhizopus oryzae & Extrapulmonary (CNS) \\
\hline 15 & Other adults & Positive & Diffuse large B-cell lymphoma & & Pulmonary \\
\hline 20 & Other adults & Negative & Meningitis & Herpes simplex virus type 1 & Pulmonary \\
\hline 21 & Other adults & Positive & Sepsis & Escherichia coli & Pulmonary \\
\hline 22 & Other adults & Negative & Sepsis & Candida glabrata & Pulmonary \\
\hline 23 & Other adults & Positive & Disseminated Kaposi's sarcoma & Human herpesvirus 8 & Pulmonary \\
\hline 24 & Other adults & Negative & Sepsis & Enterobacter spp. & Pulmonary \\
\hline 25 & Other adults & Positive & Pneumonia & Pseudomonas aeruginosa & Disseminated (lung, CNS) \\
\hline 26 & Other adults & Positive & Toxoplasmosis & Toxoplasma gondii & Disseminated (lung, liver, spleen) \\
\hline 27 & Other adults & Negative & Cardiac arrest & & Pulmonary \\
\hline 28 & Other adults & Positive & Toxoplasmosis & Toxoplasma gondii & Pulmonary \\
\hline 29 & Other adults & Positive & Sepsis & Legionella pneumophila & Disseminated (spleen, lung) \\
\hline 30 & Other adults & Positive & Meningitis & Streptococcus pneumoniae & Disseminated (lung, CNS) \\
\hline 31 & Other adults & Positive & Pneumonia & Unspecified & Pulmonary \\
\hline
\end{tabular}

$\mathrm{n}=31$. CDA: complete diagnostic autopsy; CNS: central nervous system.

pulmonary TB. Four patients showing miliary $\mathrm{TB}$ in the $\mathrm{CDA}$ were diagnosed as pulmonary $\mathrm{TB}$ or $\mathrm{TB}$ meningitis ( $\mathrm{n}=3$ and $\mathrm{n}=1$, respectively).

Clinicians specified tuberculosis as one of the diagnoses at death (or initiated TB treatment) in 36 cases. However, only 20 cases were correctly diagnosed. The sensitivity of the clinical diagnosis (including decision to treat $\mathrm{TB}$ ) for diagnosing $\mathrm{TB}$ disease at death (regardless of whether it was the $\mathrm{CoD}$ or concomitant TB) was $32.3 \%$ ( 20 out of $62,95 \%$ CI $20.9-45.3 \%$ ) with a specificity of $90.1 \%$ (95\% CI $84.1-$ $94.2 \%)$. Thus, TB disease at death remained undiagnosed in $67.7 \%$ (42 out of 62 ) of cases. By study group, the sensitivity of any clinical diagnosis to detect TB disease at death was $27.3 \%$ (three out of 11) in children, $24.4 \%$ (10 out of 41 ) in adults and $10.0 \%$ (two out of 10 ) in maternal deaths (table 4 ).

\section{Discussion}

This study, which is part of one of the largest autopsy studies conducted in sub-Saharan Africa, demonstrates the enormous burden of TB among children and adults, including maternal deaths, dying in a reference hospital in Mozambique. An even higher burden was found among HIV-positive adults and maternal cases, in whom the proportion of TB findings rose up to $51 \%$ and $39 \%$, respectively. Importantly, it also shows alarming proportions of TB disease missed by clinicians and highlights the limitations of clinical diagnosis for ascertaining TB in resource-constrained settings. In addition, the use of molecular assays (this is the first time Xpert Ultra has been used in a post mortem study) allowed M. tuberculosis to be detected in $8 \%$ (18 out of 223) of patients in whom no histological changes were identified, possibly reflecting early forms of TB. This indicates that the total burden of TB at death might be even higher than what has been reported in clinical and epidemiological and even in many autopsy studies.

A high burden of TB has also been found in some autopsy studies in the countries neighbouring Mozambique, within the context of high HIV and TB burden. In Zambia, 65\% of deaths in hospitalised 


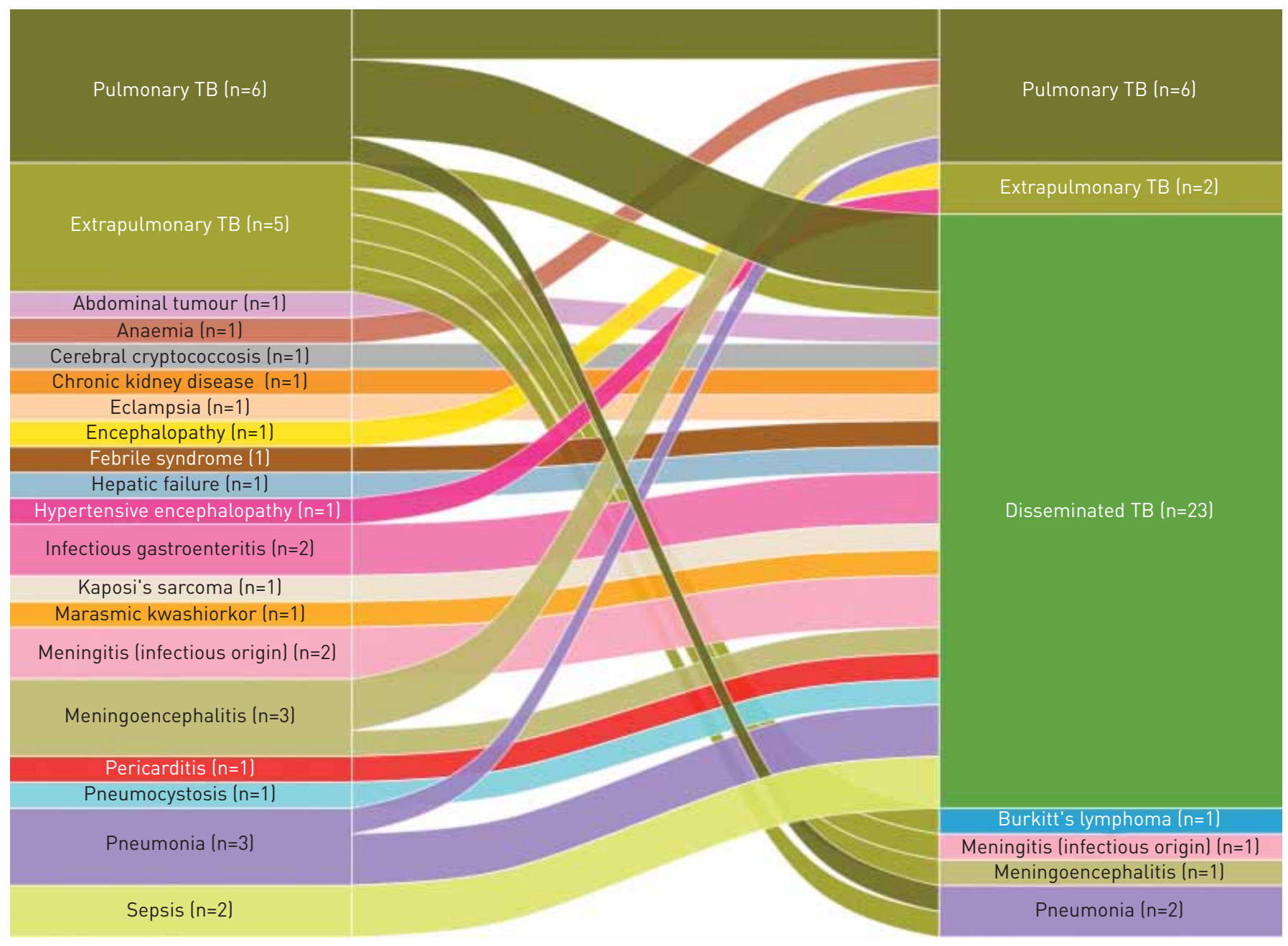

Clinical diagnosis

CDA-based diagnosis

FIGURE 2 Alluvial diagram showing cause of death (CoD) as assigned in the complete diagnostic autopsy (CDA) and per clinical diagnosis whenever a tuberculosis (TB) diagnosis was involved. The right column shows all the TB diagnoses (as CoD) in CDA ( $\mathrm{n}=31$ ) as well as the associated CDA diagnoses when clinicians assigned TB as the CoD $(n=5)$. The left column shows the clinical diagnoses of CoD when TB was specified by clinicians as well as the associated clinical diagnosis when the results of the CDA assigned TB as the CoD.

patients aged $>16$ years ( $81 \%$ HIV-positive) were due to TB, and in Kwazulu Natal, South Africa, 50\% of adult inpatient deaths aged 20-45 years had culture-confirmed TB (96\% HIV-positive) [10, 26]. A meta-analysis of autopsy studies showed that the prevalence of TB among HIV-positive adult deaths in sub-Saharan Africa was 43.2\% (95\% CI 38.0-48.3\%) [2]. We found a slightly lower percentage of cases in which $\mathrm{TB}$ was the $\mathrm{CoD}$, perhaps because of different HIV treatment status, or because in some cases TB findings were not deemed as the most likely CoD. In fact, in their meta-analysis GuPTA et al. [2] reported that in $91.4 \%$ (95\% CI $85.8-97.0 \%)$ of cases in which TB was present at death it was also the primary CoD. Interestingly, we found that $\mathrm{TB}$ was the $\mathrm{CoD}$ in $50 \%$ of cases in which $\mathrm{TB}$ disease was present at death. This considerable proportion of TB disease not causing death can largely be explained by the additional cases with concomitant TB that were captured by a detailed pathological evaluation and the use of TB-PCR and Xpert Ultra in the diagnostic algorithm, and which might have been missed in other studies using traditional diagnostic tools.

Additionally, in 18 cases with no histological evidence of TB, M. tuberculosis DNA was detected by two different molecular methods. These findings make the classification of this form of TB especially difficult, since the post mortem diagnosis of $\mathrm{TB}$ is not based on the diagnostic criteria used in living patients (sputum sample, presence of symptoms or chest radiography). It is unlikely that these findings correspond to contamination in the autopsy room, since disposable materials are used in each autopsy and the time that the tissue samples are exposed to potential airborne contamination is limited. Neither is it likely that these are false-positive cases, since DNA was detected by two different assays with different molecular 
TABLE 3 Characteristics of cases in whom the case of death was tuberculosis (TB), with concomitant TB disease at death and with Mycobacterium tuberculosis detection without histological evidence of TB

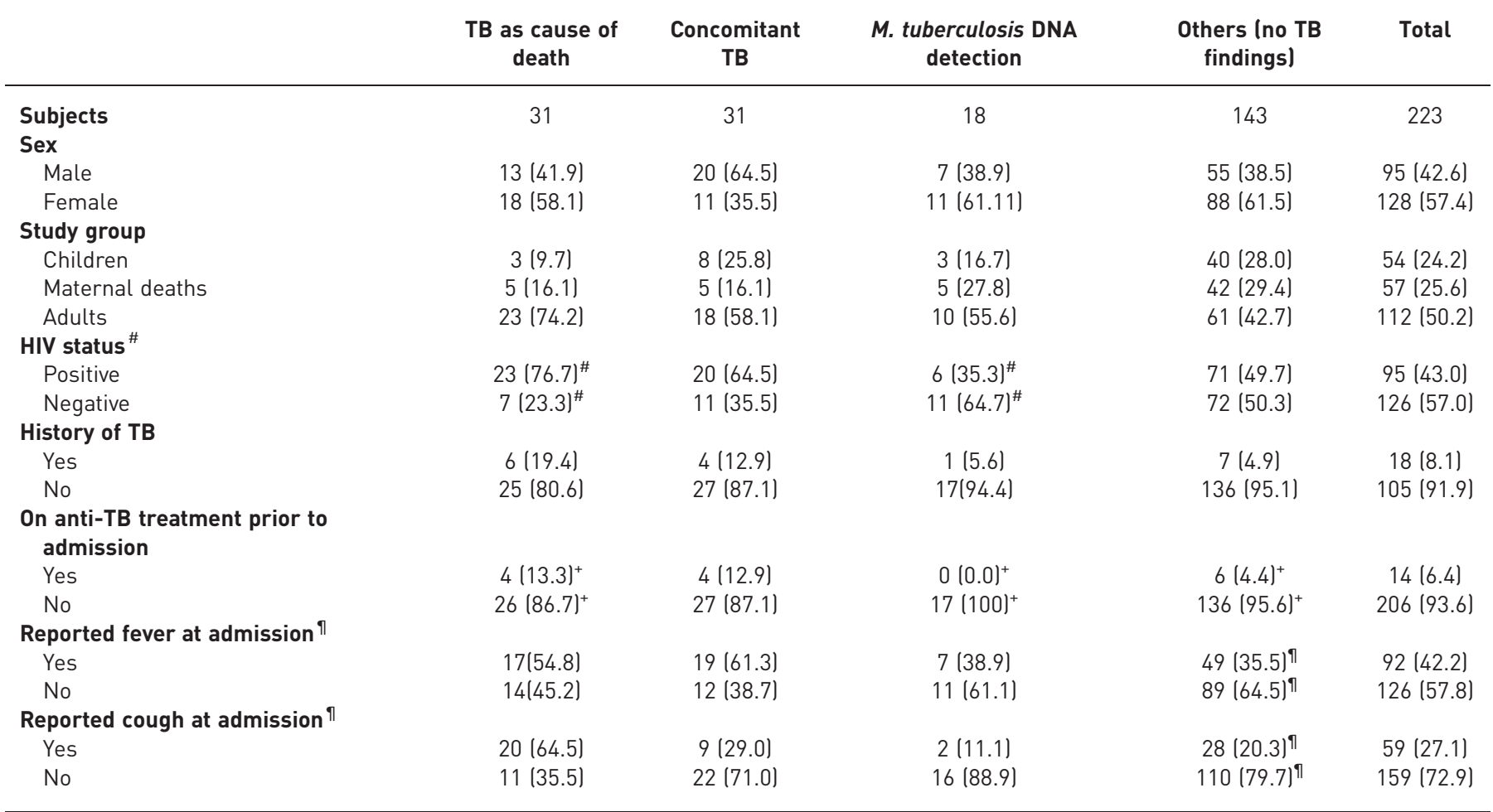

Data are presented as $\mathrm{n}$ or $\mathrm{n}(\%) .{ }^{*}$ : HIV status could not be ascertained in two cases lone in the group who died of TB, and the other among those with M. tuberculosis detection; ": fever or cough was not recorded in five cases. M. tuberculosis detection included patients in whom M. tuberculosis DNA was detected without histological evidence of $\mathrm{TB}^{+}{ }^{+}$: information was not available in three cases.

targets. These findings may represent cases of incipient TB in which small histological lesions might be present but missed, since not all the tissue from all the organs was sampled for histological analysis [27]. However, these M. tuberculosis DNA findings may also represent the increasingly controversial concept of latent TB infection. Although it has been postulated that TB infection cannot be detected through direct diagnostic methods [28], it has been suggested that it might be detected through detailed molecular studies in cases without histological evidence [29]. It has also been suggested that there could be periods in the so-called latency or unstable infection period during which M. tuberculosis replicates at a higher rate, but this replication remains self-controlled [14]. The present study suggests and supports the idea of the existence of a spectrum of TB disease, ranging from early forms of TB in which few bacilli are present (that might not necessarily develop into TB disease) to the traditional patent TB lesions (figure 3).

Clinical misclassification of $\mathrm{TB}$ was very frequent. In $>80 \%$ of $\mathrm{TB}$ identified as the $\mathrm{CoD}$ in $\mathrm{CDA}$, the clinicians failed to identify TB as the event leading to death, and overall $67 \%$ of cases with TB disease at death were missed by the clinicians. Conversely, in $45 \%$ of the cases clinicians incorrectly established TB as the main cause of death or the presence of TB disease at death. The clinicopathological discrepancies of

TABLE 4 Diagnostic performance of clinical diagnosis to determine cause of death, concomitant tuberculosis (TB) and TB disease at death (TB as cause of death + concomitant TB)

\begin{tabular}{lccc} 
& TB as cause of death & Concomitant TB & TB disease at death \\
\hline Sensitivity & $19.4(7.5-37.5)$ & $19.4(7.5-37.5)$ & $32.3(18.0-49.8)$ \\
Specificity & $97.4(94.0-99.1)$ & $90.1(85.0-93.9)$ & $90.1(87.0-95.4)$ \\
PPV & $54.5(23.4-83.3)$ & $24(9.4-45.1)$ & $55.6(25.5-64.7)$ \\
NPV & $88.2(83.1-92.2)$ & $87.4(81.9-91.7)$ & $77.5(81.7-91.6)$ \\
\hline
\end{tabular}

Data are presented as \% (95\% CI). PPV: positive predictive value; NPV: negative predictive value. 


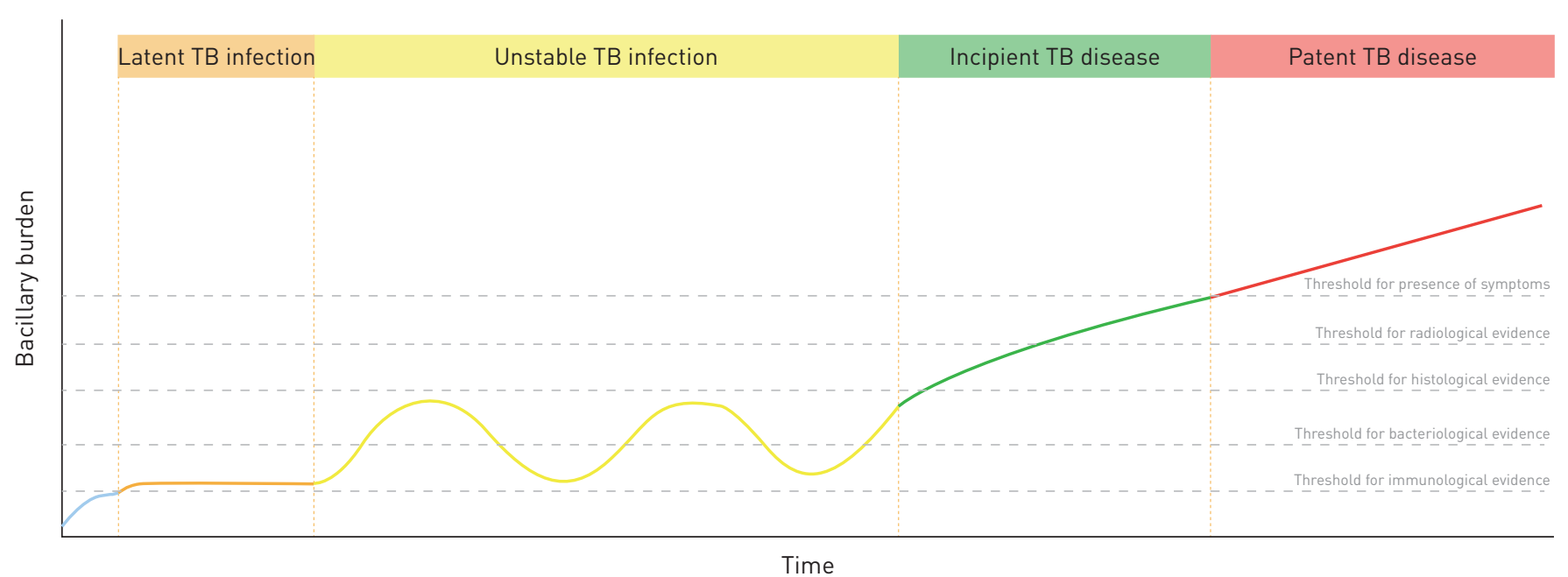

Latent TB infection
Immunological evidence
No microbiological evidence
No histological evidence
No radiological evidence

Unstable TB infection
Immunological evidence
Microbiological evidence possible
No histological evidence
No radiological evidence

Incipient TB disease
Immunological evidence
Microbiological evidence
Histological evidence
Radiological evidence possible

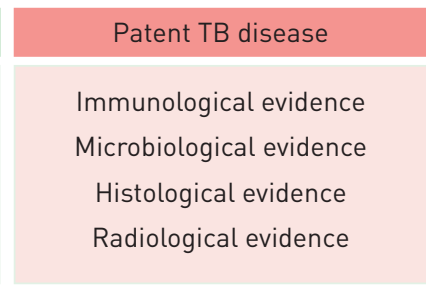

FIGURE 3 Natural history of the tuberculosis (TB) model depending on bacillary burden and the likelihood of having immunological, microbiological, histological or radiological evidence at the time of death. Adapted from a model described by Esmall et al. [14].

TB disease at death have been reported previously and have implications for patient management as well as for estimating TB mortality burden $[9,10]$. Clinical diagnosis is not a good proxy of mortality by TB, and contrarily to what it was expected, it was poor in both HIV-positive and HIV-negative cases with TB disease. Thus, it is likely that the results of verbal autopsies are similarly bad or even worse as a tool to quantify TB mortality $[12,13,30]$. Nonetheless, more studies comparing verbal autopsies with CDA are needed in order to demonstrate their usefulness as a tool for assessing TB mortality burden. It was of note that out of the 36 cases with TB findings in whom clinicians specified TB as a clinical diagnosis, only 18 were started on anti-TB treatment (prior to or during admission). Possible reasons for this may be that when the patient died, clinicians might have reconsidered the potential diagnoses of the patient and then added TB, or that patients might have been too ill or died very early during admission, thus anti-TB treatment was not initiated. Poor reporting of anti-TB treatment initiation cannot be discarded.

Our study has several limitations. First, our conclusions can only be generalised to hospitalised patients of settings with similar epidemiological characteristics and do not necessarily represent the contribution of TB to all population-based mortality in Maputo or Mozambique. The likelihood of being hospitalised depends on access to healthcare, health infrastructure, severity of disease determined by healthcare workers and severity perceived by patients and relatives, among other sociological and behavioural factors. Likewise, diagnostic discrepancies might occur less frequently in larger hospitals, thus, those found in this study (carried out at the main reference hospital of the country) might underestimate the number of clinicopathological discrepancies in general Mozambican health facilities. Second, as mentioned previously, all the organ samples were analysed histologically, but not all in their entirety, thus some cases of concomitant TB might have been missed. Third, TB culture was not contemplated in the study design, and this method could have helped to better characterise the microbiological findings. Likewise, the use of Xpert Ultra as a screening tool in all cases could have determined a higher number of confirmed cases and allowed estimation of the added yield of the use of this highly sensitive technology. Last, the quality of the clinical information may have been suboptimal in some cases of hospitalised patients with severe disease status, and their degree of consciousness might have caused information bias in some of the results. Thus, despite comprehensive evaluation of all the clinical records available, some relevant information about the medical history might have been missed by patients or relatives.

In conclusion, we found a high burden of $\mathrm{TB}$ disease at death (as $\mathrm{CoD}$ and as concomitant disease) in all groups studied. The use of highly sensitive molecular tests in CDA helped to identify cases of TB disease 
at death that would have otherwise been missed. Indeed, the results demonstrate that in our setting, clinical diagnoses miss most of the TB disease which is detected in CDA. In addition, Xpert Ultra may have the potential to identify earlier forms of $\mathrm{TB}$, before histological lesions are evident, or potentially, unstable latent TB infection.

Acknowledgements: We would like to thank the families of the deceased patients included in this study. The authors are grateful to all the members of the Dept of Pathology of Maputo Central Hospital, whose support made this study possible, and also to the staff of the Centro de Investigação em Saúde de Manhiça (CISM) for their logistic support. We specifically thank Bento Nhancale (CISM) for his invaluable support to the study. We thank Cepheid for providing the Xpert Ultra cartridges used in the study.

Support statement: The CaDMIA research project (Validation of the minimally invasive autopsy tool for cause of death investigation in developing countries) was funded by the Bill and Melinda Gates Foundation (global health grant numbers OPP1067522; Q. Bassat) (www.gatesfoundation.org/) and by the Spanish Instituto de Salud Carlos III (FIS, PI12/00757; C. Menendez) (https://portalfis.isciii.es). Data analysis was supported by the CaDMIA plus research project, funded by the Bill and Melinda Gates Foundation (global health grant numbers OPP1128001; J. Ordi) (www. gatesfoundation.org/) and the Spanish Instituto de Salud Carlos III (Acciones CIBER; C. Menendez) (www.ciberisciii.es/). ISGlobal is a member of the CERCA Programme, Generalitat de Catalunya (http://cerca.cat/en/suma/). No funding bodies had any role in study design, data collection and analysis, decision to publish, or preparation of the manuscript. This study was also partially supported by grant 2017 SGR 794 from the Agència de gestió Agència de Gestió d'Ajuts Universitaris i de Recerca (AGAUR) - Departament d'Empresa i Coneixement, Generalitat de Catalunya. Funding information for this article has been deposited with the Crossref Funder Registry.

Conflict of interest: None declared.

\section{References}

1 World Health Organization (WHO). Global Tuberculosis Report 2018. WHO, Geneva, 2018.

2 Gupta RK, Lucas SB, Fielding KL, et al. Prevalence of tuberculosis in post-mortem studies of HIV-infected adults and children in resource-limited settings. AIDS 2015; 29: 1987-2002.

3 Sugarman J, Colvin C, Moran AC, et al. Tuberculosis in pregnancy: an estimate of the global burden of disease. Lancet Glob Health 2014; 2: e710-e716.

4 World Health Organization. 2013. Date last accessed: December 4, 2017. www.who.int/tb/publications/tb_women_ factsheet.pdf?ua=1.

5 Institute for Health Metrics and Evaluation (IHME). GBD Compare. Seattle, WA, IHME, University of Washington, 2016. http://vizhub.healthdata.org/gbd-compare. Date last accessed: June 20, 2018.

6 García-Basteiro AL, López-Varela E, Respeito D, et al. High tuberculosis burden among people living with HIV in southern Mozambique. Eur Respir J 2015; 45: 547-549.

7 Uplekar M, Weil D, Lonnroth K, et al. WHO's new End TB Strategy. Lancet 2015; 385: 1799-1801.

8 García-Basteiro AL, Brew J, Williams B, et al. What is the true tuberculosis mortality burden? Differences in estimates by the World Health Organization and the Global Burden of Disease study. Int J Epidemiol 2018; 47: 1549-1560.

9 Korenromp EL, Bierrenbach AL, Williams BG, et al. The measurement and estimation of tuberculosis mortality. Int J Tuberc Lung Dis 2009; 13: 283-303.

10 Bates M, Mudenda V, Shibemba A, et al. Burden of tuberculosis at post mortem in inpatients at a tertiary referral centre in sub-Saharan Africa: a prospective descriptive autopsy study. Lancet Infect Dis 2015; 15: 544-551.

11 Ordi J, Ismail MR, Carrilho C, et al. Clinico-pathological discrepancies in the diagnosis of causes of maternal death in sub-Saharan Africa: retrospective analysis. PLoS Med 2009; 6: e1000036.

12 Maraba N, Karat AS, McCarthy K, et al. Verbal autopsy-assigned causes of death among adults being investigated for TB in South Africa. Trans R Soc Trop Med Hyg 2016; 110: 510-516.

13 Murithi S, Sitienei J, Mitchell E, et al. TB mortality measurement: comparing verbal autopsy methods to necropsy in a setting of high HIV prevalence in Siaya County, Kenya. The 46th Union World Conference on Lung Health. 2015. SOA-625-06.

14 Esmail H, Barry CE, Young DB, et al. The ongoing challenge of latent tuberculosis. Philos Trans R Soc B Biol Sci 2014; 369: 20130437.

15 Castillo P, Martínez MJ, Ussene E, et al. Validity of a minimally invasive autopsy for cause of death determination in adults in Mozambique: an observational study. PLoS Med 2016; 13: e1002171.

16 Bassat Q, Castillo P, Martínez MJ, et al. Validity of a minimally invasive autopsy tool for cause of death determination in pediatric deaths in Mozambique: an observational study. PLoS Med 2017; 14: e1002317.

17 Menendez C, Castillo P, Martínez MJ, et al. Validity of a minimally invasive autopsy for cause of death determination in stillborn babies and neonates in Mozambique: an observational study. PLoS Med 2017; 14: e1002318.

18 Castillo P, Hurtado JC, Martínez MJ, et al. Validity of a minimally invasive autopsy for cause of death determination in maternal deaths in Mozambique: an observational study. PLoS Med 2017; 14: e1002431.

19 Bassat Q, Ordi J, Vila J, et al. Development of a post-mortem procedure to reduce the uncertainty regarding causes of death in developing countries. Lancet Glob Health 2013; 1: e125-e126.

20 World Health Organization;. Maternal Mortality in 2000. Geneva, 2004. www.who.int/healthinfo/statistics/ indmaternalmortality/en/

21 Castillo P, Ussene E, Ismail MR, et al. Pathological methods applied to the investigation of causes of death in developing countries: minimally invasive autopsy approach. PLoS One 2015; 10: e0132057.

22 Martínez MJ, Massora S, Mandomando I, et al. Infectious cause of death determination using minimally invasive autopsies in developing countries. Diagn Microbiol Infect Dis 2016; 84: 80-86. 
23 Espasa M, González-Martín J, Alcaide F, et al. Direct detection in clinical samples of multiple gene mutations causing resistance of Mycobacterium tuberculosis to isoniazid and rifampicin using fluorogenic probes. J Antimicrob Chemother 2005; 55: 860-865.

24 Diedrich CR, O'Hern J, Wilkinson RJ. HIV-1 and the Mycobacterium tuberculosis granuloma: a systematic review and meta-analysis. Tuberculosis 2016; 98: 62-76.

25 World Health Organization (WHO). Medical Certification of Cause of Death, 4th Edn. WHO, Geneva, 1979. http://apps.who.int/iris/bitstream/10665/40557/1/9241560622.pdf

26 Cohen T, Murray M, Wallengren $\mathrm{K}$, et al. The prevalence and drug sensitivity of tuberculosis among patients dying in hospital in KwaZulu-Natal, South Africa: a postmortem study. PLoS Med 2010; 7: e1000296.

27 Kik S V, Schumacher S, Cirillo DM, et al. An evaluation framework for new tests that predict progression from tuberculosis infection to clinical disease. Eur Respir J 2018; 52: 1800946.

28 Getahun H, Matteelli A, Chaisson RE, et al. Latent Mycobacterium tuberculosis infection. N Engl J Med 2015; 372: 2127-2135.

29 Hernández-Pando R, Jeyanathan M, Mengistu G, et al. Persistence of DNA from Mycobacterium tuberculosis in superficially normal lung tissue during latent infection. Lancet 2000; 356: 2133-2138.

30 Karat AS, Tlali M, Fielding KL, et al. Measuring mortality due to HIV-associated tuberculosis among adults in South Africa: comparing verbal autopsy, minimally-invasive autopsy, and research data. PLoS One 2017; 12: e0174097. 